\title{
Quipu: Nudos Numéricos y Parlantes
}

\author{
Zelma Wong Torres \\ Luisa E. Salcedo Guzmán \\ Facultad de Ciencias Contables de la UNMSM \\ Lima - Perú
}

\section{RESUMEN}

Ea avance Muchos investigadores están tratando de descifrar que controlaba el Inca por intermedio de los Quipucamayocs; por las indagaciones efectuadas y siendo la mayoría de los investigadores de éste tema, Contadores Públicos; pensamos que los Quipucamayocs controlaban todo tipo de información, desde la población, tierra, producción hasta los tributos; a través de los nudos, los que representaría información numérica afras); se piensa además, que entre un nudo y otro existen espacios, los cuales indicarían la denominación del setalle de la provincia, ejército, pueblo, tiempo, además de la producción; incluso los colores de los nudos coatenían información sobre la naturaleza del objeto que era contado.

$\Xi$ Inca tenía gran cantidad de información que debía de conocer y controlar; siendo el Quipucamayoc quen zanejaba dicha información por medio de las cuentas contables, es por eso que se les exigía exactitud en la zaformación caso contrario eran aniquilados.

Palabras Clave: Quipucamayoc, Quipus, Sistema Contable Incaico, Nudos Colgantes

\section{INTRODUCCION}

El quipu se originó en el horizonte Medio o Wari, a mediados del primer milenio A.C., se utilizó para llevar la contabilidad incaica, el control de la población, posesión de tierras, el trabajo y la producción. Es un invento preincaico de cordeles colgantes hechos de lana, pelos de auquénidos o algodón, a los que teñían, de diferentes colores. Consistían en un cordón grueso, en la parte superior del cual pendían numerosos cordones verticales, delgados y con diferentes nudos, con la posibilidad de sujetar otros cordeles anexos al cordón grueso, los quipus permitían guardar información utilizando un complejo conjunto de percepciones visuales y táctiles; los nudos que estaban en ella eran de diferentes clases y colores, fue utilizado hasta el tiempo de la Colonia.

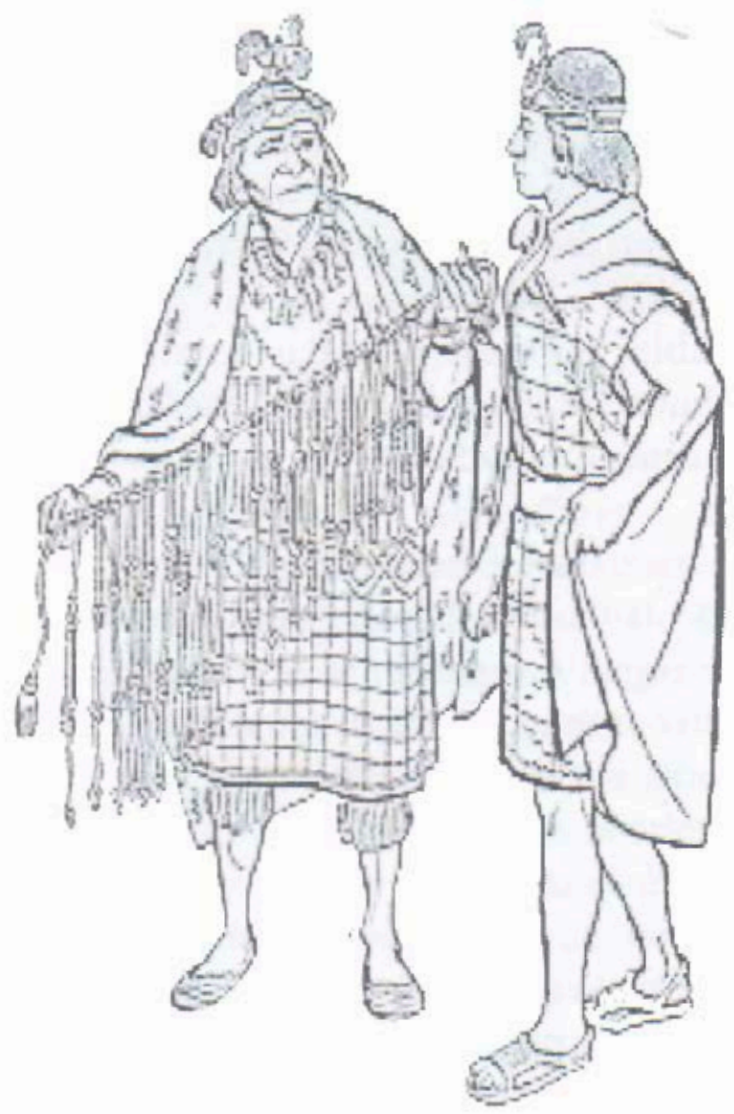


El color de los nudos, dependía de la naturaleza del objeto: amarillo para el oro, rojo para el ejército, blanco para la paz, carmesí para el Inca, el negro indicaba el tiempo, el gris para registrar acontecimientos de guerra.

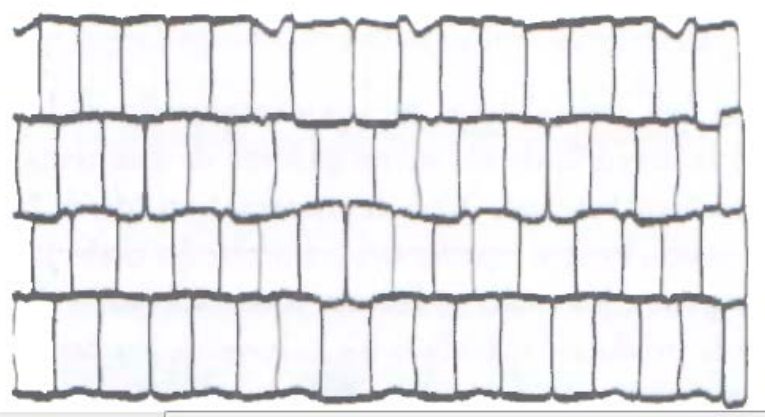

Este impresionante quipu inca, que se exhibe en el Museo Larco Herrera, muestra la complejidad alcanzada por estos artefactos contables. Probablemente haya pertenecido a un funcionario de nivel provincial. Las cuerdas, se nota, fueron agrupadas siguiendo cierto patrón repetitivo en la distribución de los colores.

\section{QUIPU}

Quipu proviene del término quechua quipuni y significa nudo. Los quipus son cordeles de algodón de fibra de camélidos (alpaca, guanacos, llamas, vicuñas), en ellos se registraban información sobre la cantidad de vasallos del Imperio, irabitantes de cada pueblo, ingresos y salidas de almacenes, tributos de los indios en especies o trabajo así como cantidad de tierras asignadas (topos).

Luego del Tercer Concilio en Lima en 1583 la Corona Española consideraba que los quipus constituían idolatrías por lo que ordenaron quemarlos, quedando de este modo, la vida de los incas en el anonimato. Cieza de León hacía referencia sobre personas expertas en descifrar pasajes históricos de los quipus. Los conquistadores no hicieron ningún esfuerzo para conocer el sistema de información inca.

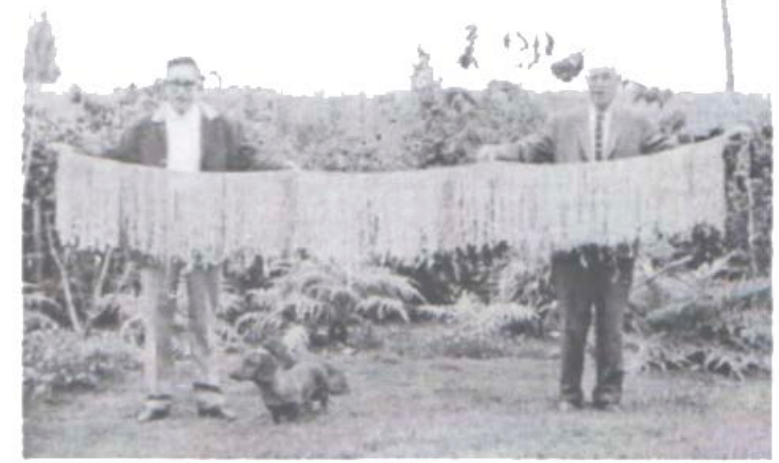

En la foto Percy Dauelsberg y el famoso arqueólogo norteamericano Junius Bird sosteniendo uno de los quipus más grandes hasta ahora conocido, proveniente de Arica, tiene $3 \mathrm{~m}$ de longitud y más de 1,500 cuerdas.

Se pensó que el quipu fue creado por el amauta Illa, en tiempo de Mayta Cápac, pero las investigaciones practicadas, determinan que el origen de los quipus es preincaico y que los incas lo hicieron suyos $y$ lo perfeccionaron para vincularlos con la contabilidad.

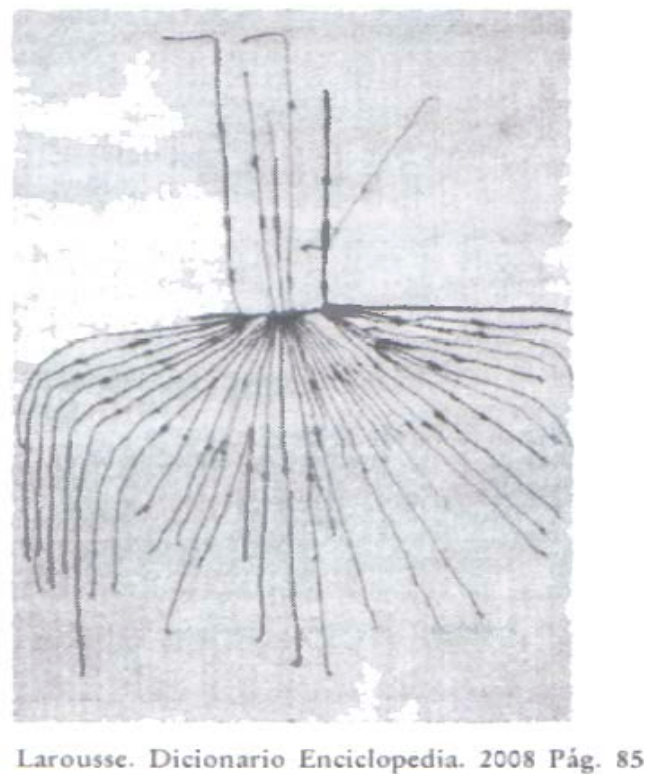

El imperio incaico estaba compartido en cuatro regiones: Chinchaysuyo, Antisuyo, Contisuyo y Collasuyo. El imperio basaba sus actividades en alianzas comerciales y en conquistas militares. El sistema de comunicaciones usado para enviar mensajes a través del chasqui, aseguraba la 
integridad del imperio en el espacio y también en el tiempo. Debido a la cantidad de información que debía ser recolectada, surgió la aparición de un nuevo funcionario llamado "Quipucamayoc" cuya labor era similar a la hoy conocida como Contadores públicos. Los Quipucamayocs estaban encargados de llevar la cuenta y dar razón de los bienes así como de la historia y acontecimientos producidos en los reinados de los diferentes Incas. Se piensa que en cada región se encontraba como mínimo 4 quipucamayocs y podían llegar a ser hasta 30, dependiendo del tamaño del pueblo, y estarían encargados de entregar cuentas tres veces al año, según lo publicado por Cieza de León; además, los Quipucamayocs, estaban encargados de informar los sucesos más importantes, los mismos que están anotados en el Quipu e informado al contador mayor, llamado "Tahuantinsuyo Runa Quipuc" quien lo revisaba e informaba al Inca. Las ciudades en las que residían, debían de proveerles de vivienda para él y su familia. Es importante mencionar que el Quipucamayoc que cometía algún error, era castigo con la muente $\mathrm{y}$ en las guerras los quipucamayocs eran aniquilados por los vencedores con la finalidad que no se guardara registro alguno de los que antes gobernaban en ese lugar.

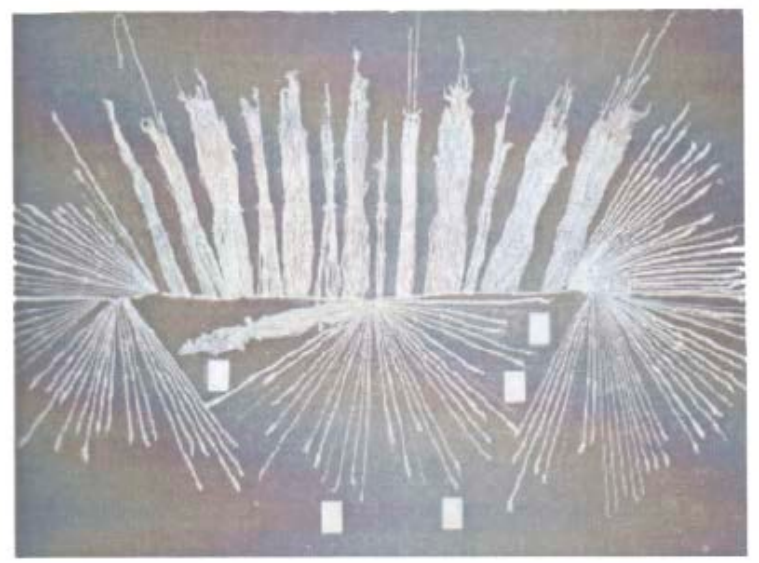

Revista Cosas $\mathrm{N}^{\circ}$ 331. Noviembre 2005. Pág. 107
La contabilidad era esencial para cobrar impuestos a la población y controlar la actividad económica. Con la ayuda de los nudos se contabilizaba los días y las fechas, de manera semejante a un calendario, quedando así registradas en los quipus. El último proceso de evolución hace que los quipus salgan del monopolio de los quipucamayoc y fueran parte de la educación de la alta sociedad inca y los futuros miembros de la nobleza.

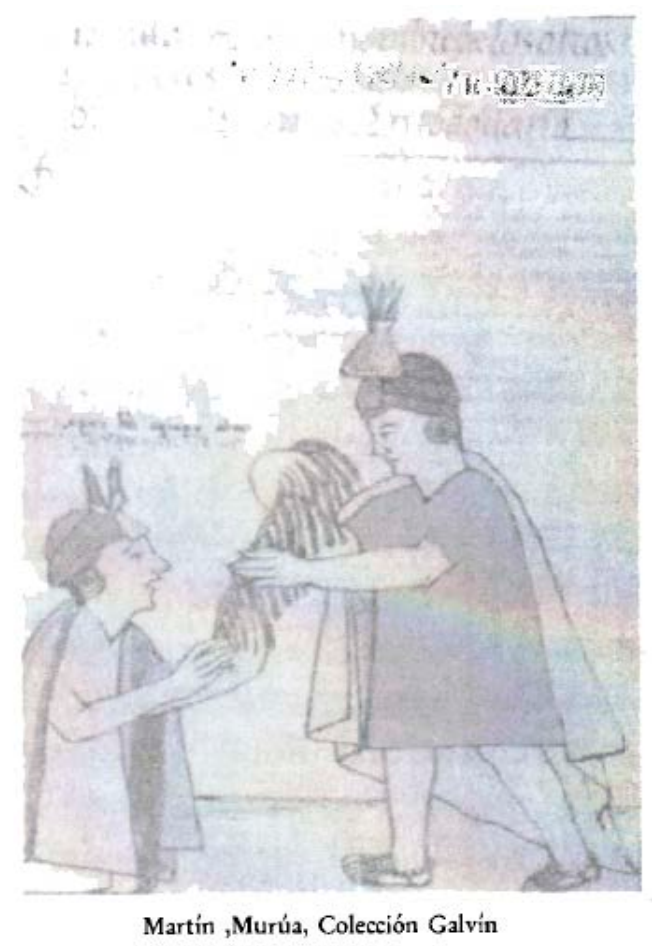

USODE LOSQUIPUS

Este método maravilloso de comunicación que se utilizó era una serie de secuencias multicolores anudadas. El color de las cuerdas era significativo y dependía de la naturaleza del objeto; mientras que los nudos eran limitados. Algunos colores tenían diversos significados dependiendo de los fines generales del Quipu. Los objetos que no se podían distinguir por color fueron alineados según calidad. Verbigracia, en el Quipu el control de inventario del armamento la lanza sería primera, por 
considerarla el arma más honorable del Inca, luego le seguían las flechas, los arcos, las hachas, etc.

La infor mación se almacenaba en un orden jerárquico determinado por las cuerdas colgantes y anexas. El color de éstas, su torsión en uno $u$ otro sentido y la ubicación y estilo de los nudos, eran los principales elementos.

Investigaciones de Leland Locke demostraron que los quipus contenían un sistema numérico decimal. En los años setenta, Marcia y Robert Asher reconstituyeron la codificación matemática contenida en los quipus.

Para las cuerdas destinadas a guardar cifras, había una ubicación y nudo específico para las unidades, decenas, centenas, etc., siguiendo un sistema decimal que concordaba con la ordenación jerárquica de las unidades productivas por cada región del imperio inca. En el extremo de una cuerda se consignaban las unidades, 1 se representa con un nudo de forma 8,2 con un nudo doble, 3 con un nudo triple, etc.; el digito "O" se representa con la ausencia del nudo en su lugar. De esa manera eran representados números relativamente grandes en la misma cuerda. Dentro de la serie de nudos eran agregados otro tipo de información como el color, el largo y ancho de la cuerda, y la misma distancia entre cuerdas $y$ entre nudos.

\section{Número 1}

Se confeccionan en forma de 8 para el enlace siguiente.

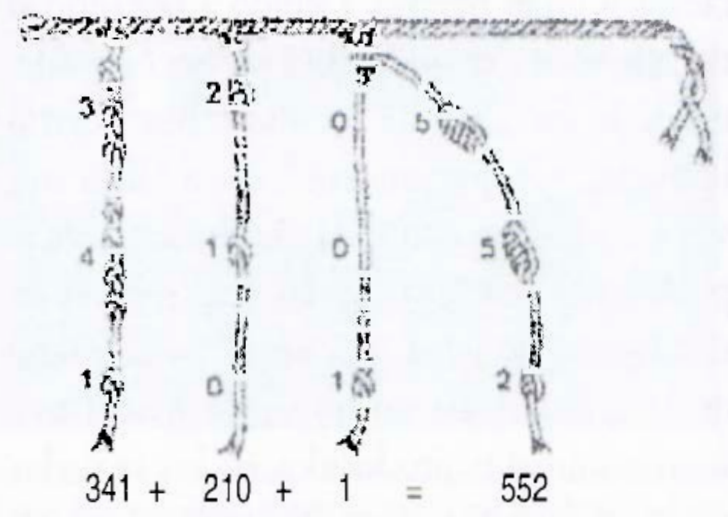

Más arriba iban nudos simples para las decenas, luego las centenas y así sucesivamente. Para el concepto de cero bastaba omitir el nudo en la ubicación correspondiente. La lectura era de derecha a izquierda del cordel principal, siguiendo cada hilo que surge. La posición y la cantidad de nudos presentaban información cuantitativa, mientras que los colores indicaban la naturaleza del objeto representado. Las operaciones matemáticas se realizaban en las yupanas y de ésta se trasladaban a los quipus. Los quipus que conocemos proceden de excavaciones arqueológicas.

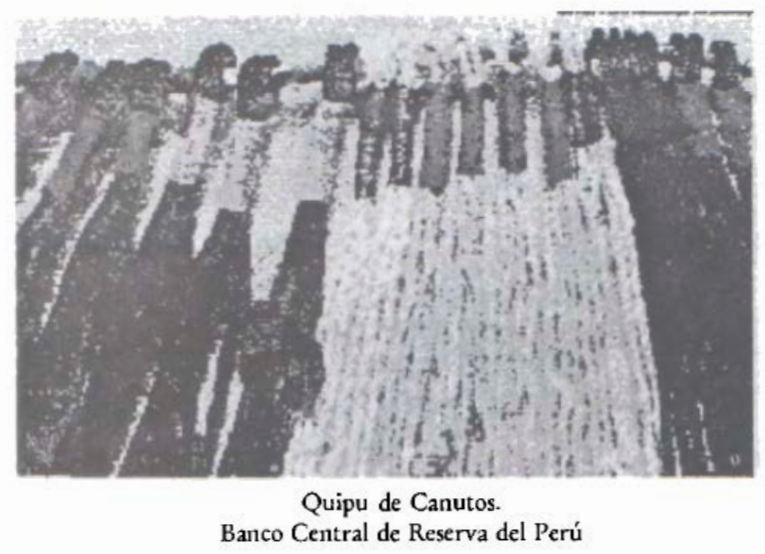

Un hilo de color gris que se ató con diez nudos pequeños, indicaba provincias sometidas. Cada uno de estos nudos tenía sujetado a él un hilo verde en el cual indicaba con los nudos el número de enemigos muertos y se agregaba pequeñas secuencias para nombrar la provincia nativa; de esta misma 
manera se agregaría una secuencia roja, para describir el número de los hombres muertos y de las provincias vencidas por el ejército imperial.

Existieron tres tıpos de quipus:

El Quipu Estadístico : Conocido y usado por todos, desde el hombre simple hasta el quipucamayoc.

La información permitıó saber las condiciones económıcas exactas de todas las regiones del imperio y las decisiones adecuadas para actuar y prevenır las catástrofes, tales como, sequía y hambre.

\section{El Quipu Ideográfico de Personas} Especializadas: Era propio de un número reducido de personas, que habían estudiado en las escuelas especiales regentados por los viejos quipucamayoc quienes dedicaban toda su vida al estudio de los nudos, obligados también a enseñar a sus hijos.

El Quipu Ideográfico de los Amautas: Estaba reservado para los amautas y quipucamayocs, por sus conocimientos eran altos funcionarios.

Es posible que los quipus no se limitaran a consignar cifras solamente sino que podían codificar hasta elaboraciones verbales, y aún las relaciones cronológicas entre diversos eventos, pero el último de los quipucamayoc, oficiales del Imperio Inca que sabían «escribir» $\mathrm{y}$ «leer» en los quipus, se llevaron sus conocimientos a la tumba.

Quipu de colores intercalados

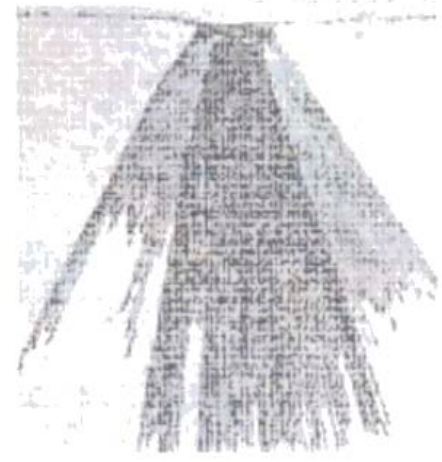

Todo científico que se ha dedicado a descifrar las cuerdas y nudos destacan que estos contienen los secretos del incanato y el mundo tiene curiosidad de descifrar el misterio.

En 1550 las Crónicas de José Da Acosta se pregunta como hacían los indios para contar y guardar información en los quipus. En 1616 el cronista Padre Martín Murúa, afirma que los quipus contienen algo más que números y cuentas, y que podría encontrarse un código de intormación narrativa. En 1920 Leland Locke demostró que los quipus tenían un significado numérico. En 1970 Marcia y Robert Ascher realizaron una exhaustiva investigación sobre el significado de los números en los quipus. El científico investigador, Gary Urton, nacido en Nuevo México en 1947, de la universidad de Harvard en Boston, escribió en el New York Times y en la revista Sciencie, causando gran sensación al publicar "String and know, theory of inca writing" (cuerdas y nudos, teorías de la escritura incaica) determinándose que los avances en desarrollar el misterio de los quipus es la llave de la puerta que llevará al pasado a muchos científicos, Urton, se ha asociado con Carrie J. Brezine para llevar adelante el "Khipu Database Proyect" clasificando toda la información que han podido recolectar del incanato.

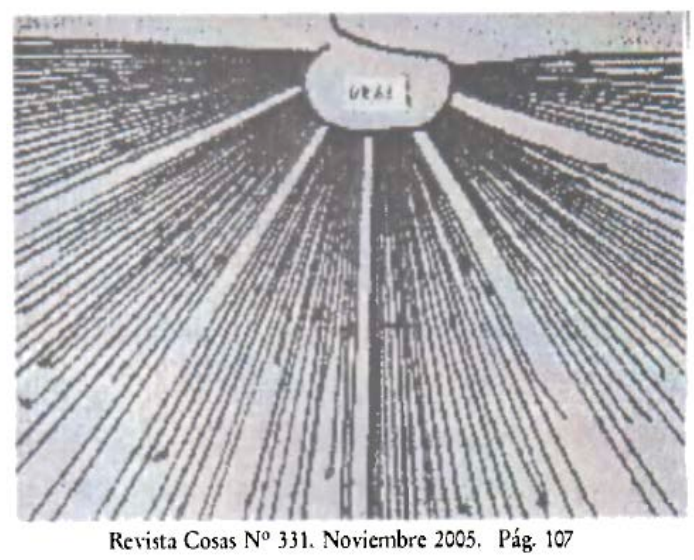


La mayoría de las grandes civilizaciones en la antigüedad desarrollaron un sistema de escritura, salvo la incaica y lo interesante de todas estas investigaciones que se esta llegando a descubrir cada vez mas sorprendentemente pues los quipus eran los libros que contenían información contable, astronómica, calendarios agrícolas, arquitectura, llegamos a un mundo increíble de los incas.

Al igual que los escribas en Egipto, los quipus estaban bajo la responsabilidad de los quipucamayocs, y cuando ellos desaparecieron, también desapareció la posibilidad de entender la infor mación registrada en los quipus.

Es desde 1990 que, Gary Urton empieza investigar sobre otros aspectos de los quipus: la dirección de cuerda, los nudos individuales y de la manera de añadir cada cuerda de la cuerda central. En los años siguentes, Urton y Brezine estudiaron las técnicas usadas en la torción y tejido se conservan todavía en algunas comunidades peruanas. Estos científicos han analizado informáticamente 21 quipus, halladas en las ruinas de Puruchuco en 1956, intentado descubrir la escritura "jeroglífica" que pudieran contener los quipus.

Los dos investigadores llegaron a la conclusión que representa el lugar de origen del quipus, es decir que es un indicador del lugar de procedencia de la información que contiene el quipu. Los quipus eran llevados de un lugar a otro, por lo cual debían de contener en el ellas la marca del lugar de origen del cual contenían la información. En los quipus hallados en Puruchuco, Urton y Brezine determinaron que una serie de 3 nudos en forma de 8 identificaban que dichos quipus pertenecían a Puruchuco. El descifrar los nudos parlantes llevará algún tiempo, entonces será posible comprender como los antiguos incas podían explicar a sacerdotes y españoles detalles históricos del Imperio Inca con simplemente ver los quipus.

Hoy se disponen de unos 600 quipus distribuidos en museos y colecciones privadas del mundo. Las investigaciones están avocadas a la hipótesis: ¿qué es lo que se controlaba en los quipus?

En la Universidad Nacional Mayor de San Marcos, en la Huaca San Marcos, se ha encontrado un quipu que data de los años 205 y $700 \mathrm{~d}$. C, está conformado por una cuerda de algodón de $11.8 \mathrm{~cm}$ de longitud de la que cuelgan 12 cuerdas de $11.5 \mathrm{~cm}$. De largo.

Por lo investigado se puede deducir que los nudos son los números y la distancia entre uno y otro sería la escritura está hipótesis se la dejamos para los futuros investigadores.

\section{REFERENCIAS}

1. Guaman Poma de Avala, Felipe, Nueva Crónica y Buen Gobierno , París 1936

2 Cieza De León, Pedro, La Crónica del Perú Madrid - España 1941

3 Porras Barrenechea, Raúl, Las Crónica del Perú 1528-1650, Volumen 2 ,Banco de Crédito del Perú - 1986

4 Historia de Tabuantinsuyo, María Rostoworoski de Diez Canseco, 6ta edición, Editorial I. E. P, Lima-Perú, 1995

5 Larousse, Diccionario Enciclopédico, Colombia 2005.

6 Rostorowski, María, Enciclopedia Editora El Comercio S.A. $2^{\circ}$ Edición, Perú 2006

7 Gertz Manero, Federico, Origen y Evolución de la Contabilidad, $5^{\circ}$ Edición, 1994

8 Millán Puentes, Régulo, La Contabilidad como Ciencia. $2^{\circ}$ Edición, 1999 